International Journal of Pure and Applied Mathematics

Volume 93 No. 1 2014, 31-43

ISSN: 1311-8080 (printed version); ISSN: 1314-3395 (on-line version)

url: http://www.ijpam.eu

doi: http://dx.doi.org/10.12732/ijpam.v93i1.4

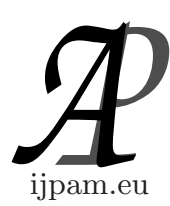

\title{
ON THE GENERALIZED NONLINEAR \\ DIAMOND HEAT KERNEL
}

\author{
Wanchak Satsanit \\ Department of Mathematics \\ Faculty of Science, Maejo University \\ Chiangmai, 50290, THAILAND
}

Abstract: In this paper, we study the nonlinear heat equation

$$
\frac{\partial}{\partial t} \triangle^{k} u(x, t)-c^{2} \diamond^{k} u(x, t)=f(x, t, u(x, t)),
$$

where $\triangle^{k}$ is the Laplacian operator iterated $k$ - times and is defined by (1.4)and $\diamond^{k}$ is the Diamond operator iterated $k$ - times and is defined by (1.2). We obtain an interesting kernel related to the nonlinear heat equation.

Key Words: Fourier transform, spectrum, diamond operator

\section{Introduction}

Consider the ultra-hyperbolic operator iterated $k$ - times defined by

$$
\square^{k}=\left(\frac{\partial^{2}}{\partial x_{1}^{2}}+\frac{\partial^{2}}{\partial x_{2}^{2}}+\cdots+\frac{\partial^{2}}{\partial x_{p}^{2}}-\frac{\partial^{2}}{\partial x_{p+1}^{2}}-\frac{\partial^{2}}{\partial x_{p+2}^{2}}-\cdots-\frac{\partial^{2}}{\partial x_{p+q}^{2}}\right)^{k},
$$

$p+q=n$. S.E. Trione[8] has shown that the generalized function $R_{2 k}(x)$ defined by (2.4) is the unique elementary solution of the operator $\square^{k}$ that is $\square^{k} R_{2 k}(x)=$ $\delta$ where $x \in \mathbb{R}^{n}$ the $n$ - dimensional Euclidean space. Also M. Aguirre Tellez[1, pp.147-149] has proved that $R_{2 k}(x)$ exists only if $n$ is odd with $p$ odd and $q$ even or only if $n$ is even with $p$ odd and $q$ odd.

Received: September 15, 2013

(C) 2014 Academic Publications, Ltd. url: www.acadpubl.eu 
In 1996, A. Kananthai[4] [2] first introduced the Diamond operator defined by

$$
\diamond^{k}=\left(\left(\sum_{i=1}^{p} \frac{\partial^{2}}{\partial x_{i}^{2}}\right)^{2}-\left(\sum_{j=p+1}^{p+q} \frac{\partial^{2}}{\partial x_{j}^{2}}\right)^{2}\right.
$$

The operator $\diamond^{k}$ can be written as the product of the operators in the form

$$
\diamond^{k}=\triangle^{k} \square^{k}=\square^{k} \triangle^{k}
$$

where $\triangle^{k}$ is the Laplacian operator iterated $k$ - times and is defined by

$$
\triangle^{k}=\left(\frac{\partial^{2}}{\partial x_{1}^{2}}+\frac{\partial^{2}}{\partial x_{2}^{2}}+\cdots+\frac{\partial^{2}}{\partial x_{n}^{2}}\right)^{k}
$$

and $\square^{k}$ is the Ultra-hyperbolic operator iterated $k$ - times and defined by(1.1).

A. Kananthai [4, p.33] has shown that the convolution $(-1)^{k} R_{2 k}^{e}(x) * R_{2 k}^{H}(x)$ is an elementary solution of the operator $\diamond^{k}$, that is

$$
\diamond^{k}\left((-1)^{k} R_{2 k}^{e}(x) * R_{2 k}^{H}(x)\right)=\delta(x),
$$

where $\delta(x)$ is Dirac-delta distribution and the function $R_{2 k}^{e}(x)$ and $R_{2 k}^{H}(v)$ are defined by (2.7) and (2.4) respectively with $\alpha=\beta=2 k, k$ is a nonnegative integer.

It is well known that for the heat equation

$$
\frac{\partial}{\partial t} u(x, t)=c^{2} \triangle u(x, t)
$$

with the initial condition

$$
u(x, 0)=f(x),
$$

where $\triangle$ is the Laplace operator and is defined by $(1.3)$ with $k=1, f(x)$ is a continuous function and $(x, t)=\left(x_{1}, x_{2}, \ldots, x_{n}, t\right) \in \mathbb{R}^{n} \times(0, \infty)$, we obtain

$$
u(x, t)=\frac{1}{\left(4 c^{2} \pi t\right)^{n / 2}} \int_{\mathbb{R}^{n}} \exp \left(-\frac{|x-y|^{2}}{4 c^{2} t}\right) f(y) d y
$$

as the solution of (1.6). The equation (1.7) can be written in the following form

$$
u(x, t)=E(x, t) * f(x)
$$

where

$$
E(x, t)=\frac{1}{\left(4 c^{2} \pi t\right)^{n / 2}} \exp \left(-\frac{|x|^{2}}{4 c^{2} t}\right)
$$


$E(x, t)$ is called the heat kernel, where $|x|^{2}=x_{1}^{2}+x_{2}^{2}+\cdots+x_{n}^{2}$ and $t>0$. Moreover, we obtain $E(x, t) \rightarrow \delta$ as $t \rightarrow 0$ where $\delta$ is the Dirac delta distribution.

Next, K. Nonlaopon and A. Kananthai [4] have studied the heat equation

$$
\frac{\partial}{\partial t} u(x, t)=c^{2} \square u(x, t),
$$

where $\square$ is the ultra-hyperbolic operator and is defined by (1.1) with $k=1$. They obtain the ultrahyperbolic heat kernel

$$
E(x, t)=\frac{i^{q}}{\left(4 c^{2} \pi t\right)^{n / 2}} \exp \left(\frac{\sum_{i=1}^{p} x_{i}^{2}-\sum_{j=p+1}^{p+q} x_{j}^{2}}{4 c^{2} t}\right),
$$

where $p+q=n$ is the dimension of the Euclidean space $\mathbb{R}^{n}$ and $i=\sqrt{-1}$.

Now, the purpose of this article is to study the nonlinear equation

$$
\frac{\partial}{\partial t} \triangle^{k} u(x, t)-c^{2} \diamond^{k} u(x, t)=f(x, t, u(x, t)),
$$

where $(x, t)=\left(x_{1}, \ldots, x_{n}, t\right) \in \mathbb{R}^{n} \times(0, \infty), t$ is a time, $c$ is a positive constant, $u(x, t)$ is an unknown function. We consider the equation (1.11) with the following conditions on $u$ and $f$ as follows

(1) $u(x, t) \in C^{(6 k)}\left(\mathbb{R}^{n}\right)$ for any $t>0$ where $C^{(6 k)}\left(\mathbb{R}^{n}\right)$ is the space of continuous function with $6 k$-derivatives.

(2) $f$ satisfies the Lipschitz condition, that is

$$
|f(x, t, u)-f(x, t, w)| \leq A|u-w|
$$

where $A$ is constant with $0<A<1$.

(3) $\int_{0}^{\infty} \int_{\mathbb{R}^{n}}|f(x, t, u(x, t))| d x d t<\infty$ for $x=\left(x_{1}, x_{2}, \ldots, x_{n}\right) \in \mathbb{R}^{n}, 0<t<$ $\infty$ and $u(x, t)$ is continuous function on $\mathbb{R}^{n} \times(0, \infty)$.

Under such conditions of $f$ and $u$ and for the spectrum of

$$
u(x, t)=(-1)^{k} R_{2 k}^{e}(x) *(E(x, t) * f(x, t, u(x, t)))
$$

as a solution of (1.11), where

$$
E(x, t)=\frac{1}{(2 \pi)^{n}} \int_{\Omega} \exp \left[c^{2} t\left(\left(\sum_{i=1}^{p} \xi_{i}^{2}\right)-\left(\sum_{j=p+1}^{p+q} \xi_{j}^{2}\right)\right)^{+i(\xi, x)}\right] d \xi
$$


and $\Omega \subset \mathbb{R}^{n}$ is the spectrum of $E(x, t)$ for any fixed $t>0$ and $(-1)^{k} R_{2}^{e}(x)$ is defined by (2.4) with $\alpha=2 k$. The convolution $(-1)^{k} R_{2}^{e}(x) * E(x, t)$ is called the Diamond Heat Kernel and all properties it will be studied in detail. Before proceeding the following definitions and concepts of needed.

\section{Preliminaries}

Definition 2.1. Let $f(x) \in \mathrm{L}_{1}\left(\mathbb{R}^{n}\right)$-the space of integrable function in $\mathbb{R}^{n}$. The Fourier transform of $f(x)$ is defined by

$$
\widehat{f}(\xi)=\frac{1}{(2 \pi)^{n / 2}} \int_{\mathbb{R}^{n}} e^{-i(\xi, x)} f(x) d x
$$

where $\xi=\left(\xi_{1}, \xi_{2}, \ldots, \xi_{n}\right)$ and $x=\left(x_{1}, x_{2}, \ldots, x_{n}\right) \in \mathbb{R}^{n},(\xi, x)=\xi_{1} x_{1}+\xi_{2} x_{2}+$ $\cdots+\xi_{n} x_{n}$ and $d x=d x_{1} d x_{2} \ldots d x_{n}$.

Also, the inverse of Fourier transform is defined by

$$
f(x)=\frac{1}{(2 \pi)^{n / 2}} \int_{\mathbb{R}^{n}} e^{i(\xi, x)} \widehat{f}(\xi) d \xi .
$$

If $f$ is a distribution with compact supports by the Equation(2.1) can be written as

$$
\widehat{f}(\xi)=\frac{1}{(2 \pi)^{n / 2}}\left\langle f(x), e^{-i(\xi, x)}\right\rangle .
$$

Definition 2.2. The spectrum of the kernel $E(x, t)$ of $(1.9)$ is the bounded support of the Fourier transform $\widehat{E(\xi, t)}$ for any fixed $t>0$.

Definition 2.3. Let $\xi=\left(\xi_{1}, \xi_{2}, \ldots, \xi_{n}\right)$ be a point in $\mathbb{R}^{n}$ and be denoted by

$\Gamma_{+}=\left\{\xi \in \mathbb{R}^{n}: \xi_{1}^{2}+\xi_{2}^{2}+\ldots+\xi_{p}^{2}-\xi_{p+1}^{2}-\xi_{p+2}^{2}-\ldots-\xi_{p+q}^{2}>0\right.$ and $\left.\xi_{1}>0\right\}$.

The set of an interior of the forward cone, and $\bar{\Gamma}_{+}$denotes the closure of $\Gamma_{+}$.

Let $\Omega$ be a spectrum of $E(x, t)$ defined by definition 2.2 for any fixed $t>0$ and $\Omega \subset \bar{\Gamma}_{+}$. Let $\widehat{E(\xi, t)}$ be the Fourier transform of $E(x, t)$ and be defined as

$$
\widehat{E(\xi, t)}= \begin{cases}\frac{1}{(2 \pi)^{n / 2}} \exp \left(c^{2} t\left(\sum_{i=1}^{p} \xi_{i}^{2}-\sum_{j=p+1}^{p+q} \xi_{j}^{2}\right)\right) & \text { for } \xi \in \Gamma_{+}, \\ 0 & \text { for } \xi \notin \Gamma_{+} .\end{cases}
$$


Definition 2.4. Let $x=\left(x_{1}, x_{2}, \ldots, x_{n}\right)$ be a point of the n-dimensional Euclidean space $\mathbb{R}^{n}$ and written as

$$
v=x_{1}^{2}+x_{2}^{2}+\ldots+x_{p}^{2}-x_{p+1}^{2}-x_{p+2}^{2}-\ldots-x_{p+q}^{2},
$$

where $p+q=n$ is the dimension of the space $\mathbb{R}^{n}$.

Let $\Gamma_{+}=\left\{x \in \mathbb{R}^{n}: x_{1}>0\right.$ and $\left.v>0\right\}$ be the interior of the forward cone and $\bar{\Gamma}_{+}$denote its closure. For any complex number $\alpha$, define the function

$$
R_{\alpha}^{H}(v)= \begin{cases}\frac{v^{\frac{\alpha-n}{L}}}{K_{\mathrm{n}}(\alpha)}, & \text { for } x \in \Gamma_{+}, \\ 0, & \text { for } x \notin \Gamma_{+},\end{cases}
$$

where the constant $K_{n}(\alpha)$ is given by the formula

$$
K_{n}(\alpha)=\frac{\pi^{\frac{n-1}{2}} \Gamma\left(\frac{2+\alpha-n}{2}\right) \Gamma\left(\frac{1-\alpha}{2}\right) \Gamma(\alpha)}{\Gamma\left(\frac{2+\alpha-p}{2}\right) \Gamma\left(\frac{p-\alpha}{2}\right)} .
$$

The function $R_{\alpha}^{H}(v)$ is called the ultra-hyperbolic kernel of Marcel Riesz and was introduced by Y. Nozaki [7].

It is well known that $R_{\alpha}^{H}(v)$ is an ordinary function if $\operatorname{Re}(\alpha) \geq n$ and it is a distribution of $\alpha$ if $\operatorname{Re}(\alpha)<n$. Let $\operatorname{supp} R_{\alpha}^{H}(v)$ denote the support of $R_{\alpha}^{H}(v)$ and suppose supp $R_{\alpha}^{H}(v) \subset \bar{\Gamma}_{+}$, that is $\operatorname{supp} R_{\alpha}^{H}(v)$ is compact.

Definition 2.5 Let $x=\left(x_{1}, x_{2}, \ldots, x_{n}\right)$ be a point of $\mathbb{R}^{n}$ and $|x|=\left(x_{1}^{2}+\right.$ $\left.x_{2}^{2}+\cdots+x_{n}^{2}\right)^{1 / 2}$. The elliptic kernel of Marcel Riesz is defined as

$$
R_{\alpha}^{e}(x)=\frac{|x|^{\alpha-n}}{W_{n}(\alpha)}
$$

where

$$
W_{n}(\alpha)=\frac{\pi^{\frac{n}{2}} 2^{\alpha} \Gamma(\alpha / 2)}{\Gamma\left(\frac{n-\alpha}{2}\right)},
$$

with $\alpha$ a complex parameter and $n$ the dimension of $\mathbb{R}^{n}$. It can be shown that $R_{-2 k}^{e}(x)=(-1)^{k} \triangle^{k} \delta(x)$ where $\triangle^{k}$ is defined by (1.4). It follows that $R_{0}^{e}(x)=\delta(x)$. The function $R_{2 k}^{e}(x)$ is called the elliptic kernel of Marcel Riesz.

Lemma 2.1. Let $L$ be the operator and defined by

$$
\mathrm{L}=\frac{\partial}{\partial t}-c^{2} \square^{k}
$$


where $\square$ is the ultra-hyperbolic defined by (1.1) for $t \in(0, \infty)$ and $c$ is a positive constant. Then we obtain

$$
E(x, t)=\frac{1}{(2 \pi)^{n}} \int_{\Omega} \exp \left[c^{2} t\left[\left(\sum_{i=1}^{p} \xi_{i}^{2}-\sum_{j=p+1}^{p+q} \xi_{j}^{2}\right)\right]+i(\xi, x)\right] d \xi
$$

as an elementary solution of (2.8)in the spectrum $\Omega \subset \mathbb{R}^{n}$ for $t>0$.

Proof. Let

$$
\mathrm{L} E(x, t)=\delta(x, t)
$$

where $E(x, t)$ is an elementary solution of operator $\mathrm{L}$ and $\delta$ is the Dirac-delta distribution. Thus

$$
\frac{\partial}{\partial t} E(x, t)-c^{2}\left(\sum_{j=p+1}^{p+q} \frac{\partial^{2}}{\partial x_{j}^{2}}-\sum_{i=1}^{p} \frac{\partial^{2}}{\partial x_{i}^{2}}\right)^{k} E(x, t)=\delta(x) \delta(t) .
$$

If we apply the Fourier transform defined by (2.1) to both sides of the equation, we obtain

$$
\frac{\partial}{\partial t} \widehat{E(\xi, t)}-c^{2}\left(\sum_{i=1}^{p} \xi_{i}^{2}-\sum_{j=p+1}^{p+q} \xi_{j}^{2}{ }^{k} \widehat{E(\xi, t)}=\frac{1}{(2 \pi)^{n / 2}} \delta(t) .\right.
$$

Thus

$$
\widehat{E(\xi, t)}=\frac{H(t)}{(2 \pi)^{n / 2}} \exp \left(c^{2} t\left(\sum_{i=1}^{p} \xi_{i}^{2}-\sum_{j=p+1}^{p+q} \xi_{j}^{2}\right)^{k},\right.
$$

where $H(t)$ is the Heaviside function. Since $H(t)=1$ for $t>0$. Therefore,

$$
\widehat{E(\xi, t)}=\frac{1}{(2 \pi)^{n / 2}} \exp \left(c^{2} t\left(\sum_{i=1}^{p} \xi_{i}^{2}-\sum_{j=p+1}^{p+q} \xi_{j}^{2}\right)\right)
$$

which has been already defined by (2.4). Thus

$$
\begin{aligned}
E(x, t) & =\frac{1}{(2 \pi)^{n / 2}} \int_{\mathbb{R}^{\mathrm{n}}} e^{i(\xi, x)} \widehat{E(\xi, t)} d \xi \\
& =\frac{1}{(2 \pi)^{n / 2}} \int_{\Omega} e^{i(\xi, x)} \widehat{E(\xi, t)} d \xi
\end{aligned}
$$


where $\Omega$ is the spectrum of $E(x, t)$. Thus from (2.4)

$$
E(x, t)=\frac{1}{(2 \pi)^{n}} \int_{\Omega} \exp \left(c^{2} t\left(\sum_{i=1}^{p} \xi_{i}^{2}-\sum_{j=p+1}^{p+q} \xi_{j}^{2}\right)^{k}+i(\xi, x)\right)^{d \xi} .
$$

Definition 2.6 We can extend $E(x, t)$ to $\mathbb{R}^{n} \times \mathbb{R}$ by setting

$$
E(x, t)=\left\{\begin{array}{lll}
\frac{1}{(2 \pi)^{n}} \int_{\Omega} \exp \left(c^{2} t\left(\sum_{i=1}^{p} \xi_{i}^{2}-\sum_{j=p+1}^{p+q} \xi_{j}^{2}\right), \text { for } t>0\right. & \\
0, & \text { for } t \leq 0 .
\end{array}\right.
$$

\section{Main Results}

Theorem 3.1. (The properties of the Heat Kernel $(-1)^{k} R_{2 k}^{e}(x) * E(x, t)$ )

(1) $(-1)^{k} R_{2 k}^{e}(x) * E(x, t)$ exists and is a tempered distribution.

(2) $(-1)^{k} R_{2 k}^{e}(x) * E(x, t) \in \mathbb{C}^{\infty}$ is the space of continuous function and infinitely differentiable.

(3) $\lim _{t \rightarrow 0}\left((-1)^{k} R_{2 k}^{e}(x) * E(x, t)\right)=(-1)^{k} R_{2 k}^{e}(x)$.

(4) $(-1)^{k} R_{2}^{e}(x) * E(x, t)$ is bounded and $|E(x, t)| \leq \frac{2^{2-n}}{\pi^{n / 2}} \frac{M(t)}{\Gamma\left(\frac{p}{2}\right) \Gamma\left(\frac{q}{2}\right)}$, for $t>$ 0 , where $M(t)$ is a function of $t$ in the spectrum $\Omega$ and $\Gamma$ denotes the Gamma function. Thus $E(x, t)$ is bounded for any fixed $t>0$.

(5) $\frac{\partial}{\partial t} \triangle^{k}\left((-1)^{k} R_{2 k}^{e}(x) * E(x, t)\right)-c^{2} \diamond^{k}\left((-1)^{k} R_{2 k}^{e}(x) * E(x, t)\right)=0$

Proof. (1) Since $E(x, t)$ and $(-1)^{k} R_{2 k}^{e}(x)$ are tempered distribution with compact support. Thus $(-1)^{k} R_{2 k}^{e}(x) * E(x, t)$ exists and is a tempered distribution.

(2) We have

$$
\frac{\partial^{n}}{\partial x^{n}}\left((-1)^{k} R_{2 k}^{e}(x) * E(x, t)\right)=(-1)^{k} R_{2 k}^{e}(x) * \frac{\partial^{n}}{\partial x^{n}} E(x, t)
$$


Since $E(x, t)$ is infinitely differentiable and $(-1)^{k} R_{2 k}^{e}(x) * E(x, t) \in \mathbb{C}^{\infty}$.

(3) By (2.4), we have

$$
E(x, t)=\frac{1}{(2 \pi)^{n}} \int_{\Omega} \exp \left(c^{2} t\left(\sum_{i=1}^{p} \xi_{i}^{2}-\sum_{j=p+1}^{p+q} \xi_{j}^{2}\right)^{k}+i(\xi, x)\right) d \xi
$$

Since $E(x, t)$ exists, then

$$
\begin{aligned}
\lim _{t \rightarrow 0} E(x, t) & =\frac{1}{(2 \pi)^{n / 2}} \int_{\mathbb{R}^{\mathrm{n}}} e^{i(\xi, x)} d \xi \\
& =\frac{1}{(2 \pi)^{n / 2}} \int_{\Omega} e^{i(\xi, x)} d \xi \\
& =\delta(x)
\end{aligned}
$$

By the continuity of the convolution,

$$
(-1)^{k} R_{2 k}^{e}(x) * E(x, t) \rightarrow(-1)^{k} R_{2 k}^{e}(x) * \delta \text { as } t \rightarrow 0 .
$$

Thus

$$
\lim _{t \rightarrow 0}\left((-1)^{k} R_{2 k}^{e}(x) * E(x, t)\right)=(-1)^{k} R_{2 k}^{e}(x) .
$$

(4) We have

$$
\begin{gathered}
E(x, t)=\frac{1}{(2 \pi)^{n}} \int_{\Omega} \exp \left(c^{2} t\left(\sum_{i=1}^{p} \xi_{i}^{2}-\sum_{j=p+1}^{p+q} \xi_{j}^{2}+i(\xi, x)\right) d \xi\right. \\
|E(x, t)| \leq \frac{1}{(2 \pi)^{n}} \int_{\Omega} \exp \left(c^{2} t\left(\sum_{i=1}^{p} \xi_{i}^{2}-\sum_{j=p+1}^{p+q} \xi_{j}^{2}\right) d \xi\right.
\end{gathered}
$$

By changing to bipolar coordinates

$$
\begin{gathered}
\xi_{1}=r \omega_{1}, \xi_{2}=r \omega_{2}, \ldots, \xi_{p}=r \omega_{p} \quad \text { and } \\
\xi_{p+1}=s \omega_{p+1}, \xi_{p+2}=s \omega_{p+2}, \ldots, \xi_{p+q}=s \omega_{p+q}
\end{gathered}
$$




$$
\begin{aligned}
& \text { where } \sum_{i=1}^{p} \omega_{i}^{2}=1 \text { and } \sum_{j=p+1}^{p+q} \omega_{j}^{2}=1 \text {. Thus } \\
& \qquad|E(x, t)| \leq \frac{1}{(2 \pi)^{n}} \int_{\Omega} \exp \left[c^{2} t\left(r^{2}-s^{2}\right)\right] r^{p-1} s^{q-1} d r d s d \Omega_{p} d \Omega_{q}
\end{aligned}
$$

where $d \xi=r^{p-1} s^{q-1} d r d s d \Omega_{p} d \Omega_{q}, d \Omega_{p}$ and $\Omega_{q}$ are the elements of the surface area of the unit sphere in $\mathbb{R}^{p}$ and $\mathbb{R}^{q}$ respectively. Since $\Omega \subset \mathbb{R}^{n}$ is the spectrum of $E(x, t)$ and we suppose $0 \leq r \leq R$ and $0 \leq s \leq T$ where $R$ and $T$ are constants, we obtain

$$
\begin{aligned}
|E(x, t)| & \leq \frac{\Omega_{p} \Omega_{q}}{(2 \pi)^{n}} \int_{0}^{R} \int_{0}^{T} \exp \left[c^{2} t\left(r^{2}-s^{2}\right)\right] r^{p-1} s^{q-1} d s d r \\
& =\frac{\Omega_{p} \Omega_{q}}{(2 \pi)^{n}} M(t) \quad \text { for any fixed } t>0 \text { in the spectrum } \Omega \\
& =\frac{2^{2-n}}{\pi^{n / 2}} \frac{M(t)}{\Gamma\left(\frac{p}{2}\right) \Gamma\left(\frac{q}{2}\right)}
\end{aligned}
$$

where

$$
M(t)=\int_{0}^{R} \int_{0}^{T} \exp \left[c^{2} t\left(r^{2}-s^{2}\right)\right] r^{p-1} s^{q-1} d s d r
$$

is a function of $t, \Omega_{p}=\frac{2 \pi^{p / 2}}{\Gamma\left(\frac{p}{2}\right)}$ and $\Omega_{q}=\frac{2 \pi^{p / 2}}{\Gamma\left(\frac{q}{2}\right)}$. Thus, for any fixed $t>0, E(x, t)$ is bounded. Since $R_{2}^{H}(x)$ is a tempered distribution with compact support, $R_{2}^{H}(x)$ is bounded. Thus $R_{2}^{H}(x) * E(x, t)$ is bounded.

(5) Since

$$
\begin{aligned}
\triangle^{k}\left((-1)^{k} R_{2 k}^{e}(x) * E(x, t)\right) & =\triangle(-1)^{k} R_{2 k}^{e}(x) * E(x, t) \\
& =\delta * E(x, t) \\
& =E(x, t)
\end{aligned}
$$

and

$$
\begin{aligned}
\diamond^{k}\left((-1)^{k} R_{2 k}^{e}(x) * E(x, t)\right) & =\square^{k} \triangle^{k}\left((-1)^{k} R_{2 k}^{e}(x) * E(x, t)\right) \\
& =\square^{k}\left(\triangle^{k}(-1)^{k} R_{2 k}^{e}(x) * E(x, t)\right) \\
& =\square^{k}(\delta * E(x, t)) \\
& =\square^{k} E(x, t)
\end{aligned}
$$


Thus

$$
\begin{aligned}
\frac{\partial}{\partial t} \triangle^{k}\left((-1)^{k} R_{2 k}^{e}(x) * E(x, t)\right. & -c^{2} \diamond^{k}\left((-1)^{k} R_{2 k}^{e}(x) * E(x, t)\right) \\
= & \frac{\partial}{\partial t} E(x, t)-c^{2} \square^{k} E(x, t) \\
= & \left(\frac{\partial}{\partial t}-c^{2} \square^{k}\right) E(x, t) \\
& =0
\end{aligned}
$$

where $E(x, t)$ is defined by $(2.9)$.

Theorem 3.2. Given the equation

$$
\frac{\partial}{\partial t} \triangle^{k} u(x, t)-c^{2} \diamond^{k} u(x, t)=f(x, t, u(x, t))
$$

for $(x, t) \in \mathbb{R}^{n} \times(0, \infty), k$ is a positive number and with the following conditions on $u$ and $f$ as follows

(1) $u(x, t)$ is the space of continuous function on $\mathbb{R}^{n} \times(0, \infty)$.

(2) f satisfies the Lipschitz condition,

$$
|f(x, t, u)-f(x, t, w)| \leq A|u-w|
$$

where $A$ is constant with $0<A<1$.

(3) $\int_{0}^{\infty} \int_{\mathbb{R}^{\mathrm{n}}}|f(x, t, u(x, t))| d x d t<\infty$ for $x=\left(x_{1}, x_{2}, \ldots, x_{n}\right) \in \mathbb{R}^{n}, 0<t<$ $\infty$ and $u(x, t)$ is continuous function on $\mathbb{R}^{n} \times(0, \infty)$.

Then we obtain the convolution

$$
u(x, t)=\left((-1) R_{2 k}^{e}(x) * E(x, t)\right) * f(x, t, u(x, t))
$$

as a solution of (3.3) for $x \in \Omega_{0}$ where $\Omega_{0}$ is a compact subset of $\mathbb{R}^{n}$ for $0 \leq t \leq T$ with $T$ is constant and $(-1) R_{2 k}^{e}(x)$ is given by (2.4) and $E(x, t)$ is given by (??).

Proof. By (3.1), we have

$$
\frac{\partial}{\partial t} \triangle^{k} u(x, t)-c^{2} \diamond^{k} u(x, t)=f(x, t, u(x, t))
$$


The above can be written in the form

$$
\frac{\partial}{\partial t} \triangle^{k} u(x, t)-c^{2} \square^{k} \triangle^{k} u(x, t)=f(x, t, u(x, t)) .
$$

Setting $v(x, t)=\triangle^{k} u(x, t)$, the equation (3.4) reduce to

$$
\frac{\partial}{\partial t} v(x, t)-c^{2} \square^{k} v(x, t)=f(x, t, u(x, t)) .
$$

Convolving both sides of (3.5) with $E(x, t)$, we obtain

$$
E(x, t) *\left[\frac{\partial}{\partial t} v(x, t)-c^{2} \square^{k} v(x, t)\right]=E(x, t) * f(x, t, u(x, t))
$$

or

$$
E(x, t) *\left[\frac{\partial}{\partial t}-c^{2} \square^{k}\right] v(x, t)=E(x, t) * f(x, t, u(x, t)) .
$$

By properties of convolution

$$
v(x, t) *\left(\frac{\partial}{\partial t}-c^{2} \square^{k}\right) E(x, t)=E(x, t) * f(x, t, u(x, t)) .
$$

SO

$$
v(x, t) * \delta(x)=E(x, t) * f(x, t, u(x, t)) .
$$

or

$$
\begin{gathered}
v(x, t)=E(x, t) * f(x, t, u(x, t)) . \\
\triangle^{k} u(x, t)=E(x, t) * f(x, t, u(x, t)) .
\end{gathered}
$$

Convolving both sides of the above equation with $(-1)^{k} R_{2 k}^{e}(x)$, we obtain

$$
\begin{aligned}
& (-1)^{k} R_{2 k}^{e}(x) * \triangle^{k} u(x, t)=(-1)^{k} R_{2 k}^{e}(x) *(E(x, t) * f(x, t, u(x, t))) . \\
& \triangle^{k}(-1)^{k} R_{2 k}^{e}(x) * u(x, t)=(-1)^{k} R_{2 k}^{e}(x) *(E(x, t) * f(x, t, u(x, t))) .
\end{aligned}
$$

By definition 2.5, we obtain the solution of (3.1).

$$
u(x, t)=(-1)^{k} R_{2 k}^{e}(x) *(E(x, t) * f(x, t, u(x, t))) .
$$

or

$$
u(x, t)=(-1)^{k} R_{2 k}^{e}(x) * \int_{-\infty}^{\infty} \int_{\mathbb{R}^{\mathrm{n}}} E(r, s) f(x-r, t-s, u(x-r, t-s)) d r d s
$$


where $E(r, s)$ is given by (2.9). We next show that $u(x, t)$ is bounded on $\mathbb{R}^{n} \times$ $(0, \infty)$. We have

$$
\begin{aligned}
|u(x, t)| & \leq(-1)^{k} R_{2 k}^{e}(x) \int_{-\infty}^{\infty} \int_{\mathbb{R}^{n}}|E(r, s)||f(x-r, t-s, u(x-r, t-s))| d r d s \\
& \leq(-1)^{k} R_{2 k}^{e}(x)|E(r, s)| N
\end{aligned}
$$

where $N=\int_{-\infty}^{\infty} \int_{\mathbb{R}^{n}}|f(x-r, t-s, u(x-r, t-s))| d r d s$. By condition (3) in Theorem 3.1 and $(-1)^{k} R_{2 k}^{e}(x)$ is a tempered distribution with compact support, we conclude $u(x, t)$ is bounded on $\mathbb{R}^{n} \times(0, \infty)$.

Lastly, we will to show that $u(x, t)$ is unique. Suppose there is another solution $w(x, t)$ of $(3.1)$. We next to show that $u(x, t)$ is unique. Let $w(x, t)$ be another solution of (2.1). Let the operator

$$
L=\frac{\partial}{\partial t} \triangle^{k}-c^{2} \diamond^{k}
$$

then (3.1) can be written in the form

$$
L u(x, t)=f(x, t, u(x, t))
$$

Thus

$$
L u(x, t)-L w(x, t)=f(x, t, u(x, t))-f(x, t, w(x, t)) .
$$

By the condition (2) of the theorem 3.1,

$$
|L u(x, t)-L w(x, t)| \leq A|u(x, t)-w(x, t)| .
$$

Let $\Omega_{0} \times(0, T]$ the compact subset of $\mathbb{R}^{n} \times[0, \infty)$ and $L: C^{(4 k)}\left(\Omega_{0}\right) \rightarrow C^{(4 k)}\left(\Omega_{0}\right)$ for $0 \leq t \leq T$.

Now $\left(C^{(2 k)}\left(\Omega_{0}\right),\|.\|.\right)$ is a Banach space where $u(x, t) \in C^{(4 k)}\left(\Omega_{0}\right)$ for $0 \leq$ $t \leq T$ and $\|.$.$\| is given by$

$$
\|u(x, t)\|=\sup _{\substack{x \in \Omega_{0} \\ 0<t \leq T}}|u(x, t)| .
$$

Then, from (3.7)with $0<A<1$, the operator $L$ is a contraction mapping on $C^{(4 k)}\left(\Omega_{0}\right)$. Since $\left(C^{(2 k)}\left(\Omega_{0}\right),\|.\|.\right)$ i a Banach space and $L: C^{(2 k)}\left(\Omega_{0}\right) \rightarrow$ $C^{(2 k)}\left(\Omega_{0}\right)$ is a contraction mapping on $C^{(2 k)}\left(\Omega_{0}\right)$, by Contraction Theorem [5, p.300], we obtain the operator $L$ has a fixe point and has uniqueness property. Thus $u(x, t)=w(x, t)$. It follows that $u(x, t)$ is unique. 


\section{Acknowledgments}

The authors would like to thank The Thailand Research Fund and Office of the Higher Education Commission and Maejo University, Chiang Mai, Thailand for financial support and also Prof.Amnuay Kananthai Department of Mathematics, Chiang Mai University for the helpful of discussion.

\section{References}

[1] M.Aguirre Tellez,The Distributional Hankel Transform of Marcel Riesz's Ultrahyperbolic kernel, Studied in Applied Mathematics 93:133-162(1994).

[2] R. Haberman, "Elementary Applied Partial Differential Equations", $2^{\text {nd }}$ Edition, Prentice-Hall International, Inc. (1983).

[3] F. John, " Partial Differential Equations", $4^{\text {th }}$ Edition, Springer-Verlag, New York, (1982).

[4] A. Kananthai.On the Solution of the n-Dimensional Diamond Operator, Applied Mathematics and Computational, Elsevier Science Inc.New York,pp. 27-37(1997).

[5] E. Kreyzig, Introductory Functional Analysis with Applications,John Willey and Sons Inc.(1978).

[6] K. Nonlaopon, A. Kananthai, On the Ultra-hyperbolic heat kernel, International of Applied Mathematics Vol.13 No.2 2003,215-225.

[7] Y. Nozaki,On Riemann-Liouville Integral of Ultrahyperbolic Type Kodai Mathematical Seminar Reports 6(2)(1964),pp.69-87.

[8] S.E. Trione. On Marcel Riesz's ultra-hyperbolic kernel Studies in Applied Mathematics, Vol. 79, Massachusetts Institute of Technology, Elsevier, Cambridge, Massachusetts, U.S.A, 1988, pp.185-191.

[9] A.H. Zemanian, Distribution Theory and Transform Analysis, McGrawhill, New York, 1965. 
